\title{
Bandagem funcional - possível recurso coadjuvante para a reabilitaçáo de pacientes hemiplégicos
}

\author{
Emilyn Borba da Silva, Daniela Tonús
}

Departamento de Terapia Ocupacional, Universidade Federal de Santa Maria - UFSM, Santa Maria, RS, Brasil

\begin{abstract}
Resumo: O Acidente Vascular Cerebral (AVC) é uma das principais causas de sequelas neurológicas, apresentando déficits motores como a hemiplegia. Os déficits motores podem gerar perda de coordenação e precisão dos movimentos necessários para um bom desempenho funcional na realização de atividades de autocuidado, lazer e produtividade, modificando significativamente o seu cotidiano. O indivíduo acometido por AVC sofre uma ruptura em suas relações familiares, afetivas, sociais e profissionais, que repercute no seu modo e estilo de vida. São diversos os métodos e as técnicas utilizadas por terapeutas ocupacionais na reabilitação desses indivíduos, assim como o uso da bandagem funcional, que são bandagens elásticas utilizadas para acometimentos musculares e articulares. Esta pesquisa teve como objetivo identificar as possíveis mudanças no desempenho ocupacional após a utilização de bandagens elásticas em indivíduos hemiplégicos acometidos de AVC. A pesquisa foi um estudo longitudinal que se ocupou dos períodos pré e pós-intervenção terapêutica ocupacional quantitativa, caracterizado como descritivo exploratório. Os resultados evidenciaram que a bandagem funcional contribuiu para o aumento da amplitude de movimento e na evolução dos componentes e áreas do desempenho ocupacional. Assim, entende-se que esse recurso é um instrumento importante para ser usado como coadjuvante no tratamento ocupacional, a fim de se obterem resultados positivos na reabilitação de hemiplégicos acometidos por AVC.
\end{abstract}

Palavras-chave: Acidente Vascular Cerebral, Atividades Cotidianas, Bandagens.

\section{Functional bandage - a possible supporting resource for the rehabilitation of hemiplegic patients}

\begin{abstract}
Cerebral vascular accident (CVA) is one of the main causes of neurological sequelae, presenting motor deficits such as hemiplegia. Motor deficits can cause loss of coordination and precision of movements necessary for a good functional performance in self-care, leisure and productivity activities, significantly changing one's routine. Individuals affected by CVA suffer a rupture in their family, affective, social and professional relationships, with repercussions in their lifestyle. Several methods and techniques are used by occupational therapists in the rehabilitation of these individuals, such as the application of functional bandages, which are elastic bandages used in muscle and joint injuries. This research aimed to identify possible changes in occupational performance after the use of functional bandages in individuals suffering from hemiplegia as a consequence of stroke. It is a longitudinal, descriptive, exploratory study of quantitative approach on pre- and post-occupational therapy intervention. The results showed that taping contributed to increased range of motion and evolution of components and areas of occupational performance. We conclude that functional bandage is an important tool to be used as an adjuvant in occupational therapy in order to obtain positive results in the rehabilitation of hemiplegic patients affected by stroke.
\end{abstract}

Keywords: Stroke, Activities of Daily Living, Bandages.

Autor para correspondência: Emilyn Borba da Silva, Departamento de Terapia Ocupacional, Universidade Federal de Santa Maria, Av. Roraima, 1000, Prédio 26, sala 1431, CEP 97105-900, Santa Maria, RS, Brasil, e-mail: emilyn.to@gmail.com

Recebido em 9/10/2013; $1^{a}$ Revisão em 12/5/2014; $2^{a}$ Revisão em 19/5/2014; Aceito em 19/6/2014. 


\section{Introdução}

Este estudo aborda uma pesquisa realizada com adultos hemiplégicos acometidos de Acidente Vascular Cerebral (AVC) e visa verificar se a utilização de bandagens elásticas influencia no desempenho ocupacional desses indivíduos.

O AVC é a terceira principal causa de morte por doença no mundo, perdendo somente para as neoplasias e outras doenças vasculares. É também responsável por um grande índice de sequelas neurológicas, tornando-se um assunto preocupante na atualidade. Dados estatísticos nacionais indicam a incidência de aproximadamente 200 mil casos de AVC anuais (ARES, 2003).

Para Nishida, Amorim e Noue (2004), o AVC é uma das principais causas das sequelas neurológicas, apresentando déficits motor, como a hemiplegia (perda total ou parcial da função motora de um hemicorpo), e podendo estar associada a outras sequelas, como afasia, alteraçóes práxicas e visoespaciais.

Os déficits motores poderão, ainda, modificar significativamente o cotidiano e a independência do indivíduo. Carvalho (2004) afirma que em um acometimento por ordem neurológica como o AVC o indivíduo sofre uma ruptura em suas relaçôes familiares, afetivas, sociais e profissionais que repercute no seu modo e estilo de vida. Segundo Souza et al. (2003), o AVC gera um grande déficit funcional, havendo perda de coordenação e precisão dos movimentos necessários para um bom desempenho na realização de atividades de vida diária (AVDs).

O desempenho ocupacional é composto por três áreas principais, de acordo com Law et al. (2009), sendo elas o autocuidado, o lazer e a produtividade. Dessa forma, atividades como as de vida diária e as atividades instrumentais, como a brincadeira, o trabalho e a participaçáo social fazem parte do desempenho ocupacional e do cotidiano singular de cada indivíduo.

Higienizar-se, trabalhar, atender ao telefone, cozinhar, jogar cartas com amigos são alguns exemplos dessas atividades que podem apresentar-se com prioridades diferentes no dia a dia de cada pessoa. De Faria (2007) afirma que as avaliaçôes da Terapia Ocupacional têm como foco a execução das atividades valorizadas pelo indivíduo, identificando assim a problemática que pode estar interferindo no desempenho ocupacional. Para Law et al. (2009), a abordagem integrada e equilibrada das três áreas, autocuidado, lazer e produtividade, é a essência do desempenho ocupacional.
Assim, tal pesquisa pôde analisar as áreas do desempenho antes e após a utilização de bandagens funcionais, priorizando as três áreas já citadas, observando também aspectos ligados à amplitude de movimento articular dos indivíduos pesquisados e identificando a importância desse equipamento como coadjuvante no tratamento ocupacional. Motivaram este estudo a probabilidade de o uso das bandagens contribuir para a ampliação das possibilidades de recursos da Terapia Ocupacional na área de reabilitação física e a escassez de trabalhos referentes a esse tema na literatura.

\section{Acidente Vascular Cerebral: Possíveis consequências e influências no cotidiano}

O índice de acometidos de AVC cresce à medida que aumenta a expectativa de vida e que os indivíduos mudam seu estilo de vida. Consequentemente, aumenta a populaçáo mais idosa, considerada o grupo de maior acometimento (ABRAMCZUK; VILELA, 2009). Pesquisas indicam que o estresse, a vida tumultuada, a alimentaçáo errada, o sobrepeso, o tabagismo, entre tantos outros aspectos podem ser responsáveis por acometimentos de ordem neurológica como o AVC (BRINGEL, 2002).

Conforme Gillen (2005) e Ares (2003), idade, hipertensão, doenças cardíacas, diabetes, tabagismo, uso excessivo de álcool, drogas ilícitas, obesidade, o uso de contraceptivos orais, histórico familiar, fatores do estilo de vida, como, por exemplo, obesidade, estresse e sedentarismo, são fatores de riscos associados às causas do AVC.

$\mathrm{Na}$ vida adulta, a maioria dos indivíduos encontra-se em uma fase produtiva, realizando-se profissionalmente, inserindo-se no mercado de trabalho, constituindo família e, portanto, passando por uma fase de grandes realizaçóes e de muito empenho. As doenças incapacitantes, principalmente as de ordem neurológica, dificultam o desempenho ocupacional, de modo que atividades importantes para cada sujeito, especialmente na fase adulta, tão produtiva, não conseguem ser realizadas satisfatoriamente.

Albuquerque (2003) cita algumas das intervençóes realizadas por terapeutas ocupacionais na reabilitação pós-AVC como, por exemplo, a estimulação da sensibilidade do membro acometido, as orientaçóes para o domicílio, as atividades bilaterais e a estimulação cognitiva e psicossocial. São diversos os métodos e as técnicas utilizados na reabilitação 
física por terapeutas ocupacionais para melhorar o desempenho funcional desses indivíduos.

De acordo com a American Occupational Therapy Association (1974), a Terapia Ocupacional, para facilitar o desempenho ocupacional, pode incluir métodos de tratamento elaborados para a correção ou compensação de déficits em áreas de desempenho. Consideram-se estratégias de correçâo o fortalecimento muscular, a reeducaçáo sensorial e o treinamento cognitivo, bem como as estratégias de compensaçáo e o uso de dispositivos tecnológicos, como órteses e próteses.

Dentre os métodos e as técnicas utilizados podem-se citar as bandagens funcionais, também chamadas de Kinesio Taping, como um novo método para a reabilitação de pessoas com acometimentos musculares e articulares. Seu principal objetivo é oferecer estímulos proprioceptivos com o intuito de ativar as fibras musculares de acordo com a necessidade de cada indivíduo.

\section{Bandagem funcional: Ação coadjuvante na reabilitação}

O método Kinesio Taping foi criado por um quiroprata japonês chamado Kenzo Kase que, em suas pesquisas, desenvolveu bandagens elásticas com sistema inovador capaz de auxiliar, por meio de um apoio externo, a funçáo dos tecidos, sem limitar a sua ação. As bandagens elásticas suportam de forma seletiva músculos e grupos musculares. Esse material que deu nome ao método foi utilizado e aplicado no início dos anos 1990 no Japão (MATOS, 2002).

A bandagem elástica é $100 \%$ composta de acrílico hipoalérgico, não contendo quaisquer substâncias químicas, e é extensível a até cerca de $140 \%$ do seu comprimento inicial. Sua espessura e peso são similares ao da pele e a característica de ser à prova d'água permite que as bandagens mantenham-se aderentes à pele por até cinco dias.

A bandagem promove uma tensáo constante e permite a função muscular sem limitar o indivíduo em sua mobilidade. O objetivo desse dispositivo é desencadear estímulos sensoriais e mecânicos através dos mecanoreceptores presentes na derme e na epiderme. Existem quatro técnicas de aplicação: muscular, ligamentosa, corretiva e linfática (MATOS, 2002; KUMBRINK, 2011).

Acredita-se que um dispositivo como a bandagem, que auxilia o posicionamento prolongado de um segmento, seja ele uma articulação, um músculo ou um tendáo, pode ser considerado um posicionador funcional. Sendo assim, é considerado como um tipo de órtese temporária ou dispositivo de auxílio tecnológico, como as tecnologias assistivas, já que seu objetivo é melhorar a funcionalidade do membro.

Segundo a Portaria SAS/MS n. 661, de 2 de dezembro de 2010 (BRASIL, 2010), os profissionais de Terapia Ocupacional e Fisioterapia têm o direito e a competência de prescrever "[...] órteses, próteses e materiais especiais não relacionados ao ato cirúrgico". Segundo Jaraczewska e Long (2006), as bandagens elásticas têm um histórico de uso por terapeutas ocupacionais, fisioterapeutas, educadores físicos e outros profissionais de saúde para alcançar a melhoria em diversos tratamentos.

\section{Materiais e métodos}

A pesquisa foi um estudo longitudinal que se ocupou dos períodos pré e pós-intervenção terapêutica ocupacional quantitativa devido aos instrumentos de coleta de dados utilizados, caracterizados como descritivos-exploratórios. Gil (1991) afirma que a pesquisa descritiva utiliza técnicas padronizadas de coleta de dados, tais como questionários e instrumentos padronizados de avaliação.

A pesquisa exploratória, para Köche (2009), descreve e caracteriza as variáveis que se quer conhecer e pode ser quantitativa ou qualitativa. A investigação quantitativa atua em níveis de realidade e tem como objetivo trazer à luz dados, indicadores e tendências observáveis (SERAPIONI, 2000).

\subsection{Amostra e critérios de inclusão e exclusão}

Foram selecionados cinco participantes acometidos por AVC isquêmico, sendo quatro do sexo feminino e um do sexo masculino. Quatro pacientes tinham entre 43 e 52 anos e tempo de acometimento de seis meses a dois anos, e uma paciente, idosa, tinha 82 anos e 12 anos de acometimento. Quanto ao lado do acometimento, quatro participantes tiveram o lado contralateral hemiplégico e apenas um o lado dominante. Todos apresentavam um padráo hipotônico e dificuldades de realizar movimentaçâo ativa, como, por exemplo, preensão palmar e extensão da mão e dos dedos para pegar e soltar um objeto, levar a mão ao rosto ou acima da cabeça. Além disso, apresentavam o ombro em posição de depressão devido à hipotonia muscular.

Os critérios de inclusão utilizados nesta pesquisa foram: hemiplégicos acometidos de AVC usuários do Serviço de Fisioterapia da APAE de Santa Maria, de ambos os gêneros, acima de 18 anos, de todas 
as raças ou credos e que aceitassem participar desta pesquisa, estando dispostos a usar as bandagens. Assim, os critérios de exclusão foram pessoas que não estavam disponíveis a usar a bandagem, não concordaram com o Termo de Consentimento Livre e Esclarecido (TCLE), pessoas com idade abaixo de 18 anos, isto é, pessoas que não estavam adequadas aos critérios de inclusão. Pessoas com feridas abertas e que apresentavam alergia ao acrílico também foram excluídas da pesquisa.

\subsection{Local}

Os participantes foram pessoas provenientes do Serviço de Fisioterapia da Associação de Pais e Amigos dos Excepcionais (APAE) do Município de Santa Maria, RS.

No primeiro contato com a instituição onde a pesquisa foi realizada fez-se a leitura do TCLE e, após a sua assinatura, marcou-se o próximo encontro, no qual foram realizadas as primeiras avaliaçóes, a COPM e a goniometria, conforme previsto no projeto. No terceiro encontro iniciaram-se as aplicações das bandagens funcionais nos membros superiores dos participantes.

\subsection{Instrumentos de coletas de dados}

A coleta dos dados desta pesquisa utilizou como instrumento a Medida Canadense de Desempenho Ocupacional (COPM) que, de acordo com Law (2009), trata-se de um protocolo específico da Terapia Ocupacional que visa avaliar, por meio da perspectiva do indivíduo, sua satisfaçáo em relação ao seu desempenho ocupacional nas diversas áreas das atividades do cotidiano, isto é, autocuidado, lazer e produtividade.

Além disso, utilizou-se a goniometria, técnica empregada para avaliar a amplitude de movimento (ADM) de uma articulação. Marques (2008) afirma que a goniometria é uma parte importante da avaliação e diz respeito à medida de ângulos articulares presentes nas articulaçôes.

\subsection{Procedimentos para a coleta de dados}

Primeiramente, a pele foi higienizada com o Gel PRE-K-GEL, que contém antialérgico específico para a preparação da pele antes das bandagens. Após a higienização, realizou-se a medição para o recorte das bandagens.

Em todos os participantes foram aplicadas as bandagens funcionais nas formas das letras I, Y ou $\mathrm{X}, \mathrm{da}$ inserção proximal até a distal dos músculos extensores dos dedos, extensores radial longo e curto do carpo, bíceps, deltóide e supraespinhal. Todas as aplicaçôes foram em base neutra e extensão prévia do membro do tipo tonificante, caracterizadas por tonificar a musculatura, e tiveram como objetivo a melhoria da amplitude do movimento ativo.

O estudo contou com 10 encontros semanais que foram realizados de março a maio de 2013. Além das aplicaçôes das bandagens funcionais também foram realizadas massagens nas articulaçôes das falanges e apresentadas orientações para as AVDs. No decorrer dos atendimentos, um participante desistiu do tratamento devido à dificuldade de locomoção e dependência da família para o transporte, totalizando, desse modo, quatro participantes na pesquisa. Todos os participantes realizavam concomitantemente fisioterapia.

\subsection{Procedimentos para análise dos dados}

Os resultados coletados foram transcritos, analisados e interpretados pela pesquisadora através da perspectiva quantitativa e descritiva do conteúdo. De acordo com Silva et al. (2005), a análise de conteúdo está relacionada à interpretação e definição das açôes, condiçôes e situaçóes, ou seja, ao contexto em que a pesquisa foi realizada. Assim, a ação humana entrelaça-se às questóes relacionadas a palavras, gestos e a outros fatores simbólicos que não podem ser quantificados mas interpretados de acordo com a singularidade de cada contexto. Para a análise quantitativa foi utilizada a média dos resultados dos participantes e a comparação da evolução antes e após as aplicaçóes e avaliaçóes. Os dados foram apresentados em formato de gráficos para melhor compreensão do leitor.

\subsection{Aspectos éticos}

A presente pesquisa, intitulada "O uso de Órteses, Próteses e Tecnologias Assistivas para a inclusão de pessoas com deficiências físicas e sensoriais", foi conduzida de acordo com os padróes éticos exigidos, tendo passado e sido autorizada pelo Comitê de Ética e Pesquisa em Seres Humanos, CAAE 10273813.7.0000.5346. O presente estudo é subprojeto da referida pesquisa.

\section{Resultados e discussão}

Após a análise dos dados, pôde-se evidenciar que todos participantes evoluíram em relação à $\mathrm{ADM}$ na avaliação de goniometria, conforme pode ser visto 
nas Figuras 1 e 2. Na avaliação da COPM, por meio da qual são avaliados o desempenho e a satisfação quanto à realização das atividades do cotidiano, conforme a Figura 3, três participantes evoluíram e um manteve a pontuação quanto ao desempenho. Já em relação à satisfação, dois evoluíram e dois mantiveram a mesma pontuação.

As reavaliaçôes de goniometria foram realizadas nas articulaçôes dos dedos, do punho, do cotovelo e do ombro. A Figura 1 representa a demonstração da média dos participantes com o escore inicial e final da goniometria da flexão da falange proximal, média e distal do primeiro ao quinto dedo do membro acometido.

O gráfico apresenta os dados em graus e apresenta a evolução quanto à amplitude de movimento articular, com exceçáo de uma participante, que apresentou regressão no primeiro dedo, influenciando, assim, a média de evolução dos quatro participantes. No entanto, não foi encontrada na literatura uma explicação que pudesse embasar os resultados e, por ser a única ocorrência de regressão, o fato não foi considerado significativo para o estudo.

A Figura 2 representa a evolução da média em graus dos participantes nas articulaçóes do punho, cotovelo e ombro. Percebe-se que o desvio radial apresentado náo evoluiu devido aos participantes já terem alcançado a ADM dentro dos padrôes normais antes das aplicaçóes. Na pesquisa de Santos et al. (2010) quanto à aplicação de bandagem funcional em três participantes acometidos por AVC com subluxação de ombro pôde-se observar que, após as aplicaçóes de bandagens, houve diminuição da subluxação inferior de ombro, melhora na simetria postural e diminuição das compensaçôes na execução dos movimentos, além da obtenção de aumento na ADM em movimentos ativos do ombro. Também no estudo de caso de Campos, Palma e Santos (2007) em que a bandagem funcional foi utilizada para o tratamento de tendinite e tenossinovite, concluiu-se que a utilização da bandagem para contensão muscular produziu efeitos positivos no ganho da $\mathrm{ADM}$ e que, na maioria dos eixos de movimento estudados, houve melhora significativa no processo álgico.

Assim, a pesquisa em questão também demonstrou que a aplicação das bandagens funcionais oferece resultados satisfatórios quanto à amplitude de movimento de todas as articulaçóes do membro superior. Ainda, tais dados podem influenciar de forma positiva a funcionalidade dos participantes, conforme será demonstrado na COPM.
Foi possível verificar um significativo aumento de amplitude de movimento nas referidas articulaçóes. Uma participante que realizava apenas movimentação passiva de flexão de ombro, após as aplicaçôes, passou a realizar fibrilaçóes na musculatura. Segundo Bobath (2001), músculos aparentemente hipotônicos conseguem realizar contração de modo efetivo a partir de um estímulo sensorial adequado e forte.

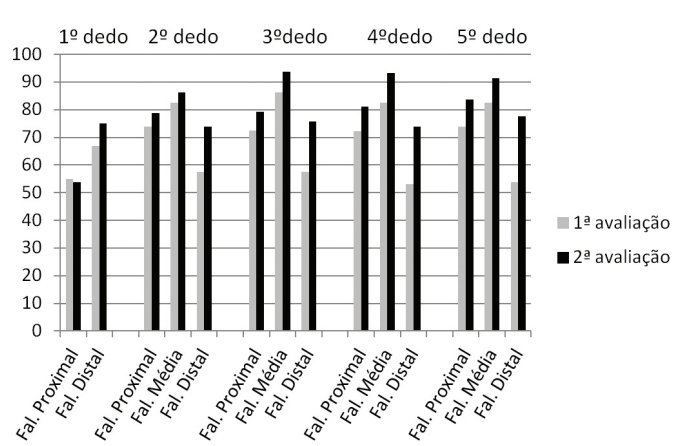

Figura 1. Demonstração em graus da média de amplitude do movimento de flexão inicial (cinza) e final (preto) da falange proximal, média e distal do $1^{\circ}$ ao $5^{\circ}$ dedo.

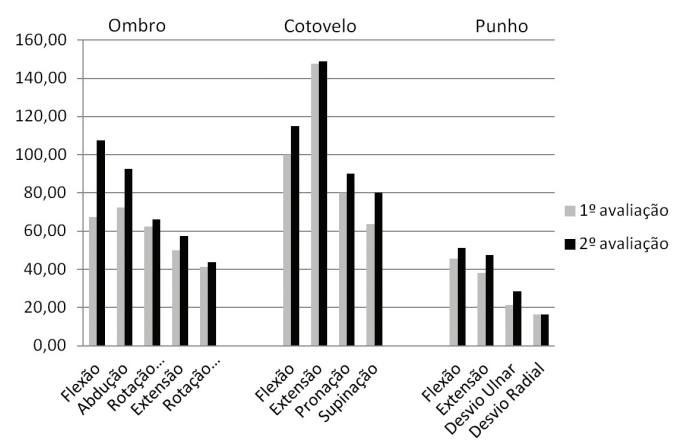

Figura 2. Demonstração da amplitude de movimento inicial (cinza) e final (preto) do ombro, cotovelo e punho.

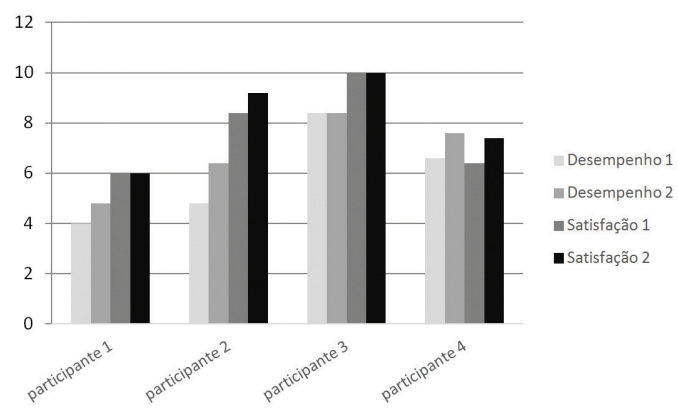

Figura 3. Escore inicial e final obtido pelos quatro participantes no desempenho e satisfação, respectivamente, na Medida Canadense de Desempenho Ocupacional. 
Os resultados da pesquisa de Paulino (2010) evidenciaram aumento ou conservação da ADM inicial tanto ativa quanto passiva, comparada com a ADM após aplicação da técnica de Kinesio Taping, no movimento de dorsiflexão do tornozelo de indivíduos que sofreram AVC.

Observa-se que a bandagem funcional realiza um estímulo constante na musculatura devido à sua aderência à pele por até cinco dias. O estímulo proprioceptivo levou os participantes a prestarem maior atençáo e cuidado ao membro acometido, estimulando-os, ainda, a não o negligenciarem. Hack (2009) cita a bandagem elástica como facilitadora na percepção corporal devido à sua ação direta sobre os receptores cutâneos.

$\mathrm{Na}$ avaliação da COPM, tendo como base a avaliação inicial, conforme pode ser visto na Figura 3, verificou-se que apenas um participante náo sofreu alteração no escore de desempenho e satisfação, enquanto que o restante variou positivamente.

A partir da análise dos gráficos pôde-se observar que dois participantes sofreram uma variação nos escores de desempenho entre 0,8 e 1,6 e que o restante manteve a nota inicial, obtendo a média de 0,85 no escore de desempenho. Na pontuação de satisfaçáo, o escore variou entre 0,8 e 1 ponto, sendo que dois participantes mantiveram a pontuaçáo inicial, obtendo a média de 0,45 ponto de variação. Desse modo, a média dos participantes de desempenho 1 em escore foi 5,95; a dos de desempenho 2 foi, em escore, 6,8; e a média da pontuação referente à satisfação 1 foi, em escore, 7,7; e a referente à satisfação 2, 8,15. Verificou-se uma variação da pontuação dos participantes em relação ao escore de desempenho (escore: 4 a 8,4) e de satisfação (escore: 6 a 10). Essa variação pode ser justificada pela composição dos participantes da amostra, que contemplou adultos de diferentes faixas etárias e amplo tempo de acometimento do AVC.

A COPM, que tem como objetivo mensurar a autopercepção do paciente em relação ao seu desempenho ocupacional, apresentou resultado positivo, afirmando-se assim que a bandagem proporcionou uma maior percepção do membro nas atividades, ou um maior envolvimento do paciente em seu processo de reabilitação.

Os escores obtidos na reavaliação após a aplicação das bandagens permitiram constatar que não houve variação acima de dois pontos para satisfação e desempenho. Para Law et al. (2009), a variaçáo de dois ou mais pontos na COPM é considerada clinicamente significativa. Andolfato e Mariotti (2009) afirmam que as razóes para a evolução nas pontuaçóes podem ser interpretadas a partir de três suposiçôes: as intervençóes da Terapia Ocupacional foram eficientes, o uso da COPM possibilitou maior envolvimento e motivação do indivíduo no processo terapêutico. Já para Law et al. (2009) essa evolução nas pontuaçôes significa que o estado geral de saúde do indivíduo melhorou.

Pôde-se interpretar que, devido às intervençôes da Terapia Ocupacional e à utilização das bandagens, a avaliação da goniometria resultou em ganho de amplitude de movimento em todos os participantes. Também foi possível perceber maior envolvimento dos participantes na reabilitação, pois eles demonstraram realizar tentativas de atividades bimanuais no cotidiano, evitando, assim, a negligência do lado plégico, estimulando o membro lesionado e, consequentemente, melhorando os componentes do desempenho ocupacional. Entretanto, observou-se que não houve tempo suficiente para que o estudo pudesse beneficiar os participantes com uma melhora mais significativa no desempenho ocupacional e na satisfação.

$\mathrm{Na}$ pesquisa realizada por Salles, Almeida e Ferreira (2012), que apresentou um estudo de caso de um paciente pós-AVE utilizando como recurso o uso do Kinesio Taping no ombro hemiparético, foram encontrados aumentos no ganho funcional de $50 \%$ no autocuidado, de $52,63 \%$ na mobilidade e de $66,66 \%$ na locomoção; e que a ADM de flexão apresentou um ganho funcional de $28 \%$ de flexão e a ADM de abdução, de 27,33\% de abdução. Esses dados se assemelham aos dados deste estudo.

Os participantes apresentavam o mesmo padrão hipotônico, no entanto a amostra continha uma ampla diferença de tempo de acometimento. Foi identificada uma melhora atenuante nos pacientes com menos de dois anos de acometimento, do que nos pacientes que já haviam sido acometidos há 12 anos, sugerindo que esse fato pode estar relacionado ao período de regeneração neural.

Acredita-se que seja de fundamental importância o enfoque dos profissionais da saúde em métodos diferenciados de reabilitação, contribuindo, conforme Sampaio, Mancini e Fonseca (2002), para a amenização do impacto que determinada condiçáo de saúde traz para a vida pessoal, familiar e social do indivíduo.

\section{Conclusão}

Neste estudo pôde-se verificar que a bandagem elástica contribuiu para o aumento da amplitude de movimento e para a melhora nos componentes do 
desempenho ocupacional. $\mathrm{O}$ recurso das bandagens elásticas na reabilitação física é um instrumento importante usado como coadjuvante no tratamento ocupacional, sendo considerado de baixo custo em comparação ao custo de materiais para a confecção de órteses.

$O$ fato de os participantes não serem pessoas jovens e de o tempo de acometimento ser tardio, o que interfere na regeneração neural, pode ter influenciado nos resultados. É importante destacar a necessidade de aplicaçáo em tempo breve de acometimento para melhor identificar o efeito da terapêutica adotada. Apesar da idade dos participantes ser influente nos resultados e de a abordagem ter sido com aplicaçôes de bandagem por um curto período de tempo, o estudo apresentou resultado positivo, o que indica uma grande influência na reabilitação de pacientes hemiplégicos acometidos de AVC.

Por ser uma abordagem recente, há poucos estudos sobre a eficácia da bandagem elástica, sendo de suma importância a realizaçáo de mais pesquisas sobre a eficácia desse método na área de reabilitação de pacientes acometidos por AVC, as quais possam comprovar seus efeitos e benefícios. $\mathrm{Na}$ área de Terapia Ocupacional, em que são poucas as bibliografias sobre o uso desse material, fazem-se necessárias maior investigação e mais publicaçóes que possam contribuir para o aprimoramento das técnicas da profissáo.

\section{Referências}

ABRAMCZUK, B.; VILELA, E. A luta contra o AVC no Brasil. Revista Eletrônica de Jornalismo Científico, Sáo Paulo, 10 jun. 2009. Disponível em: <http://www. comciencia.br/>. Acesso em: $15 \mathrm{dez} .2012$.

ALBUQUERQUE, S. H. Acidente Vascular Encefálico. In: TEIXEIRA, E. et al. Terapia Ocupacional na reabilitação fisica. São Paulo: Roca, 2003. p. 333-378.

AMERICAN OCCUPATIONAL THERAPY ASSOCIATION - AOTA. A curriculum guide for occupational therapy educators. Rockville: AOTA, 1974.

ANDOLFATO, C.; MARIOTTI, M. C. Avaliaçấo do paciente em hemodiálise por meio da medida canadense de desempenho ocupacional. Revista de Terapia Ocupacional da USP, São Paulo, v. 20, n. 1, p. 1-7, 2009.

ARES, M. J. J. Acidente Vascular Encefálico. In: TEIXEIRA E. et al. Terapia Ocupacional na reabilitação física. São Paulo: Roca, 2003. p. 3-16.

BOBATH, B. Hemiplegia em adultos: avaliação e tratamento. 3. ed. São Paulo: Manole, 2001.

BRASIL. Ministério da Saúde. Secretaria de Atenção à Saúde. Portaria no 661, de 2 de dezembro de 2010. Diário Oficial da União, Poder Executivo, Brasília, DF, 3 dez. 2010. Disponível em: <http://bvsms.saude.gov.br/ bvs/saudelegis/sas/2010/prt0661_02_12_2010.html>. Acesso em: 15 dez. 2012.

BRINGEL, A. Inimigo silencioso. Revista Super Saudável, Sáo Paulo, v. 68, n. 9, 2002. p. 4-7.

CARVAlHO, L. M. G. Terapia Ocupacional na reabilitaçáo de pacientes neurológicos adultos. In: DE CARLO, M. M. R. P.; LUZO, M. C. M. Terapia Ocupacional: reabilitaçáo física e contextos hospitalares. São Paulo: Roca, 2004. p. 200-232.

CAMPOS, L.; PALMA, R.; SANTOS, R. A. Efeitos da bandagem funcional em paciente com tendinite e tenossinovite. In: JORNADA CIENTÍFICA FIB, 2., 2007, Bauru. Anais... Bauru: FIB, 2007. p. 5. (v. 1).

DE FARIA, I. Neurologia adulto. In: CAVALCANTI, A.; GALVÃO, C. Terapia Ocupacional: fundamentação e prática. Rio de Janeiro: Guanabara Koogan, 2007. p. 188-204

GIL, A. C. Como elaborar projetos de pesquisa. 3. ed. São Paulo: Atlas, 1991

GILLEN, G. Acidente Vascular Encefálico. In: PEDRETTI, L. W.; EARLY, M. B. Terapia Ocupacional: capacidades práticas para disfunçôes físicas. São Paulo: Roca, 2005. p. 675-703.

HACK, L. F. O uso de bandagens funcionais para estimulação da musculatura respiratória de pacientes asmáticos. 2009. 67 f. Monografia (Trabalho de Conclusão de Curso em Fisioterapia)-Centro Universitário Feevale, Novo Hamburgo, 2009.

JARACZEWSKA, E.; LONG, C. Kinesio taping in stroke: improving functional use of the upper extremity in hemiplegia. Topics in Stroke Rehabilitation, Illinois, v. 13, n. 3, p. 31-42, 2006. PMid:16987790. http://dx.doi. org/10.1310/33KA-XYE3-QWJB-WGT6

KOCHE, J. C. Fundamento de metodologia cientifica: teoria da ciência e iniciação a pesquisa. Petrópolis: Vozes, 2009. KUMBRINK, B. K. Taping springer. Berlin: Auflage, 2011. LAW, M. et al. Medida Canadense de Desempenho Ocupacional (COMP). Belo Horizonte: UFMG, 2009.

MARQUES, A. P. Manual de goniometria. 2. ed. Barueri: Manole, 2008.

MATOS, N. Kinesio taping: conceitos e aplicaçôes no mundo do desporto. Revista Training, Lisboa, n. p. 10-12, 2002.

NISHIDA, A. P.; AMORIM, M. Z. M.; NOUE, M. M. E. A. Índice de Barthel e o estado funcional de pacientes pós acidente vascular cerebral em programa de fisioterapia. Revista Salusvita, Bauru, v. 23, n. 3, p. 467-477, 2004.

PAULINO, G. L. C. Efeito do Kinesio taping no movimento de dorsiflexão do tornozelo em pacientes com acidente vascular encefálico da clínica de fisioterapia da UNESC: análise eletromiográfica. 2010. 72 f. Monografia (Trabalho de Conclusão de Curso em Fisioterapia)-Universidade do Extremo Sul Catarinense, Criciúma, 2010.

SALLES, F. L. P.; ALMEIDA, R. L. D.; FERREIRA, D. M. O uso do kineiso tape associado a facilitaçấo neuromuscular proprioceptiva na melhora do controle 
motor no ombro hemiparético. Revista Brasileira de Reabilitação e Atividade Física, Vitória, v. 1, n. 1, p. 42-47, 2012.

SAMPAIO, R. F.; MANCINI, M. C.; FONSECA, S. T. Produção científica e atuação profissional: aspectos que limitam essa integração na fisioterapia e na terapia ocupacional. Revista Brasileira de Fisioterapia, Sáo Carlos, v. 6, n. 3, p. 113-118, 2002.

SANTOS, J. C. C. et al. A influência da Kinesio Taping no tratamento da subluxação de ombro no Acidente Vascular Cerebral. Revista Neurociências, São Paulo, v. 18, n. 3, p. 335-340, 2010.
SERAPIONI, M. Métodos qualitativos e quantitativos na pesquisa social em saúde: algumas estratégias para a integração. Ciência \& Saúde Coletiva. Rio de Janeiro, RJ. v. 5, n. 1, p. 187-192, 2000.

SILVA, C. R. et al. O uso da análise de conteúdo como uma ferramenta para a pesquisa qualitativa: descrição e aplicação do método. Organizaçôes Rurais \& Agroindustriais, Lavras, v. 7, n. 1, p. 70-81, 2005.

SOUZA, S. R. S. et al. Reabilitação funcional para membros superiores pós-acidente vascular encefálico. Fisioterapia Brasil, Rio de Janeiro, v. 4, n. 3, p. 195-199, 2003.

\section{Contribuição dos Autores}

Emilyn Borba da Silva foi responsável pela concepção do texto manuscrito, elaboração, coleta e análise dos dados. Daniela Tonús foi responsável pela orientaçáo da pesquisa e revisão do artigo. 